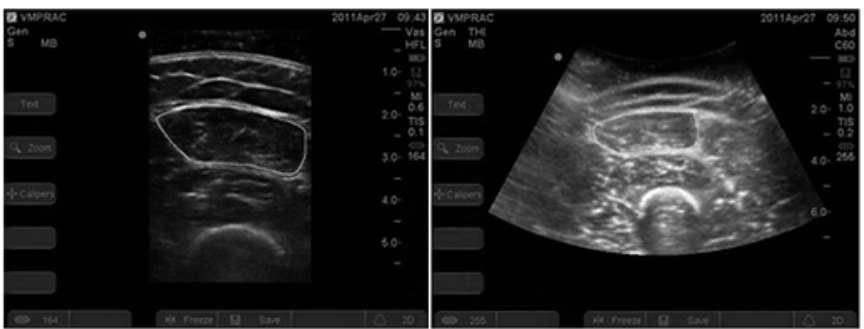

Abstract P132 Figure 1 RFcsa taken with a linear probe (image on left) and curvilinear probe (image on right) in the same individual at 2/3.

Conclusion These data demonstrate that both linear and curvilinear probes can be used to acquire accurate RFcsa measurements. Furthermore, "splicing" the images from the LUP, when a CUP is not available is a justified method to assess RFcsa. This method should be considered for RFcsa image acquisition in obese patients.

\section{P133 LUNG CLEARANCE INDEX IS A REPRODUCIBLE AND SENSITIVE MEASURE OF AIRWAYS DISEASE IN BRONCHIECTASIS}

doi:10.1136/thoraxjnl-2011-201054c.133

${ }^{1} \mathrm{~S}$ Rowan, ${ }^{1} \mathrm{~J}$ Bradley, ${ }^{1} \mathrm{M}$ Ennis, ${ }^{2} \mathrm{~A}$ Horsley, ${ }^{3} \mathrm{~N}$ Bell, ${ }^{4} \mathrm{P}$ Gustafsson, ${ }^{1} \mathrm{~J} \mathrm{~S}$ Elborn. ${ }^{1}$ Queens University Belfast, Belfast, $N$ Ireland; ${ }^{2}$ University of Manchester, Manchester, UK; ${ }^{3}$ Bristol Royal Infirmary, Bristol, UK; ${ }^{4}$ Central Hospital, Skovde, Sweden

Introduction Lung clearance index (LCI) is a measure of ventilation in homogeneity derived from multiple breath washout (MBW). Although $\mathrm{FEV}_{1}$ is commonly used to assess severity of airway disease and response to therapy, it is insensitive to small airways disease and is often within normal range in bronchiectasis (BE) not caused by Cystic Fibrosis (CF) until disease is well established. In CF, LCI is more sensitive than $\mathrm{FEV}_{1}$ in detecting airways abnormalities and is currently used as an outcome measure in clinical trials. In $\mathrm{BE}$, there is a need to find a sensitive outcome measure that is responsive to interventions, particularly in those with mild disease.

Objective To assess within and between visit repeatability of $\mathrm{LCI}$ and determine the relationship between $\mathrm{FEV}_{1}$ and LCI in stable BE.

Methods Inclusion criteria: HRCT diagnosis of BE within the last 5 years; clinically stable (no infective symptoms for $>4$ weeks); no genetic or clinical features of CF. Participants attended for two visits, 2 weeks apart. At each visit they performed MBW in triplicate, using $0.2 \%$ sulphur hexafluoride and a modified Innocor device. LCI was derived from the mean of at least 2 acceptable washouts. Spirometry was performed to ATS/ERS standards.

Results 14 patients $(8 \mathrm{M} / 6 \mathrm{~F})$ attended for two visits. The mean (SD) age was 60.5 (15.4)yrs. Mean (SD) $\mathrm{FEV}_{1} \%$ predicted was 87.1 (18.6), range (44-117). Mean (SD) LCI was 9.4 (2.0) on visit 1 and 9.4 (1.9) on visit 2 (normal $<7.5$ ). The intra-visit coefficient of variation (CV) was $4.7 \%$ (3 measures). Between visit repeatability of LCI was 0.54 (SD of variance between visits). LCI negatively correlated with $\mathrm{FEV}_{1}(\mathrm{r}=-0.69, \mathrm{p}<0.001)$. Sensitivity of LCI and $\mathrm{FEV}_{1}$ for the diagnosis of bronchiectasis by CT was $71 \%$ and $29 \%$ respectively.

Conclusions This is the first report of LCI in non-CF BE. LCI is a more sensitive test of lung function than $\mathrm{FEV}_{1}$ and is abnormal in

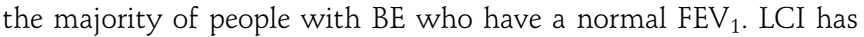
good intra-visit and between visit repeatability. Across a range of $\mathrm{FEV}_{1}$ there is a strong relationship between LCI and $\mathrm{FEV}_{1}$

\section{P134 VALIDATING STRUCTURED LIGHT PLETHYSMOGRAPHY (SLP) AS A NON-INVASIVE METHOD OF MEASURING LUNG FUNCTION WHEN COMPARED TO SPIROMETRY}

doi:10.1136/thoraxjnl-2011-201054c.134

${ }^{1} \mathrm{~S}$ Alimohamed, ${ }^{1} \mathrm{~K}$ Prosser, ${ }^{1} \mathrm{C}$ Weerasuriya, ${ }^{2} \mathrm{R}$ lles, ${ }^{3} \mathrm{~J}$ Cameron, ${ }^{3} \mathrm{~J}$ Lasenby, ${ }^{4} \mathrm{C}$ Fogarty. ${ }^{1}$ School of Clinical Medicine, University of Cambridge, Addenbrookes Hospital, Cambridge, UK; ${ }^{2}$ Department of Respiratory Paediatrics, Cambridge University Hospitals, Addenbrookes Hospital, Cambridge, UK; ${ }^{3}$ Signal Processing and Communications Laboratory, Department of Engineering, University of Cambridge, Cambridge, UK; ${ }^{4}$ Department of Statistics, Harvard University, Cambridge, Massachusetts, USA

Background Structured Light Plethysmography (SLP) is a recently developed technology for non-invasive and entirely non-contact monitoring of lung (respiratory) function. The system projects a structured light grid onto the thoraco-abdominal surface of the subject, which is imaged by two cameras giving a dynamic $3 \mathrm{D}$ reconstruction of the surface as the subject breathes. From this data we can infer changes in chest/abdomen volume over time, allowing us to extract parameters and generate curves (eg, Volume-Time, Flow-Time, Flow-Volume curves) directly comparable to conventional spirometry. SLP therefore hopes to provide an inexpensive replacement for conventional spirometry, which is an invasive methodology unusable in a number of patient classes (eg, neonates, young children, intensive care patients etc). This study tests the validity of SLP in terms of reproducibility, repeatability and position dependence, as compared to conventional spirometry (Pneumatach); by comparison of ventilation parameters extracted from both technologies.

Methods SLP and Pneumatach spirometry were used simultaneously to capture 120 datasets from 10 randomly chosen adult subjects. Each complete dataset contained tidal breathing and forced expiratory manoeuvres, in both sitting and standing positions. Operatordependence (reproducibility) was tested by collecting data sets from each subject using three different operators. Repeatability was tested by collecting the data from each subject once, and then again after a 40 min break. Tidal Inspiratory Time (TI) parameters were extracted from the results and the data analysed using the paired Student t test.

Results There was no significant difference between TI values obtained from SLP compared to conventional spirometry throughout the study $(n=120 ; p=0.8556)$. SLP comparisons of pooled mean TI before, and after a 40 min break were not significant (1.5589 vs $1.5595 ; \mathrm{p}=0.9938$ ); similarly, readings in different positions (sitting or standing) were not significantly different. SLP comparison of all three operator pairs (1vs2, 1vs3 and 2vs3), were not significant ( $\mathrm{p}=0.7361, \mathrm{p}=0.9765, \mathrm{p}=0.7343$, respectively).

Conclusions SLP measurements are not operator, time or position dependant. Therefore SLP shows a high degree of reproducibility and repeatability; and represents a promising, viable and non-invasive alternative to conventional spirometry.

\section{P135 MAXIMUM INSPIRATORY FLOW MEASURED DIRECTLY WITH AN INSPIRATORY FLOW METRE COMPARED WITH MEASUREMENTS FROM FLOW VOLUME LOOP TRACES}

doi:10.1136/thoraxjnl-2011-201054c.135

M C P Apps, M Skipper, J Skipper, S Basford, V Bryant, J Saines, T Andrews. Queen's Hospital, Romford, UK

Introduction Low Maximum Inspiratory Flow is characteristic of patients with muscle weakness, Laryngeal dysfunction, or Extrathoracic airway obstruction. We have been exploring the use of an Inspiratory Flow Metre (In Check Dial- Clement Clarke International) to help in the identification of those patients who need more detailed investigation. 